\title{
An Anticorrosive Magnesium/Carbon Nanotube Composite
}

\author{
M. Endo*, T. Hayashi, I. Itoh, Y. A. Kim, D. Shimamoto, H. Muramatsu, Y. Shimizu
}

Faculty of Engineering, Shinshu University, 4-17-1 Wakasato, Nagano 380-8553, Japan

\section{S. Morimoto}

Nagano Techno-foundation, 1-18-1 Wakasato, Nagano 380-0928, Japan

\section{Terrones}

Advanced Materials Department, IPICyT, Camino a la Presa San Jose 2055, Lomas 4a seccion, 78216 San Luis Potosi, SLP, Mexico

S. Iinou

Showa Denko K.K., 13-9, Shiba Daimon 1-Chome, Minato-ku, Tokyo 105-8518 Japan

S. Koide

Nissei Plastic Industrial Co, Ltd., 2110 Minamijo, Sakaki-machi, Nagano 389-0693, Japan

\begin{abstract}
Here we report a drastically improved anticorrosive characteristic of magnesium alloy composites with the introduction of multi-walled carbon nanotubes. Highly depressed corrosion of nanotube-filled magnesium composite in salt water is due to the formation of stable oxide films along the grain boundaries of magnesium. Our results indicate that carbon nanotube acted as effective multifunctional filler to improve both mechanical and anti-corrosive performances of magnesium alloy.
\end{abstract}

Keywords: Carbon nanotubes; Magnesium alloy; Powder processing; Corrosion property

Corresponding author: Tel: +81-26-269-5201; fax: +81-26-269-5208

E-mail: endo@endomoribu.shinshu-u.ac.jp 
Magnesium is known to be the lightest metal that can be applied to structural components, and despite its corrosive nature, its alloys are widely used in automobiles components, electronic appliances, sporting goods, etc. However, a drawback in processing magnesium alloys consists in the necessity of applying special coatings (or plating techniques) in order to avoid the corrosion of the magnesium alloys. ${ }^{1}$ For these reasons their commercial uses have been limited. In the past, it has been demonstrated that magnesium alloys could be mechanically reinforced with micro particles of graphite and carbon fibers. Unfortunately, the latter magnesium-composites lowered the corrosion resistivity due to galvanic interactions ${ }^{2,3}$ and therefore, special treatments were needed to slow down or inhibit the corrosion. ${ }^{4}$

In this letter, we show that the strategic incorporation of multi-walled carbon nanotubes (MWNTs) as filler in the magnesium alloy matrix, could drastically improve the corrosion resistance. Our results clearly demonstrate that when the filler becomes nanometer-sized, the composite becomes more corrosion resistant, thus contrasting previous results using macroscopic graphite fibers and graphite micro-particles ${ }^{5,6}$. In addition, several significant studies showed that the introduction of carbon nanotubes resulted in lightweight composites with enhanced mechanical strength. ${ }^{7-11}$ In particular, we proved that the short, linear and well-ordered MWNTs directly contributed to 25 and $11 \%$ increases in the elastic modulus and tensile strength of magnesium alloy composites, respectively. ${ }^{11}$ From these results, we envisage that MWNTs-filled magnesium composites are possibly the best candidates for constructing lightweight materials for automobiles, always considering an energy-efficient environment. We believe that nanotube-filled magnesium alloy composites could replace aluminum alloys in some commercial markets and will boost the replacement of engineering plastics where the components with lightweight, hardness and mechanical strength are required.

We prepared MWNTs-filled magnesium alloy composites by a powder-powder blending and the subsequent vacuum hot-press and extruding processes. ${ }^{11}$ Fine-grained powder (average grain size: $100 \mu \mathrm{m}$ ) magnesium alloy (AZ91D) was prepared using tri-axial vibrating-type ball miller (TKMAC-1200L, $10 \mathrm{~mm} \phi$ zirconia ball, argon atmosphere) at $800 \mathrm{rpm}$ for 6 hours. MWNTs with diameter around 80-100 nm, commercially available from Showa Denko Co. Ltd. ${ }^{12}$, was prepared by the catalytic chemical vapor deposition method ${ }^{13}$ followed by the high-temperature heat treatment in argon at $2773 \mathrm{~K}$. The MWNTs were mechanically shortened to $\sim 5 \mu \mathrm{m}$ long with a high-speed blade cutting machine. 0,1 and $5 \mathrm{wt} \%$ shortened MWNTs were mixed with magnesium alloy powder in the ball miller (1 mm $\phi$ zirconia ball) at $800 \mathrm{rpm}$ for $5 \mathrm{hrs}$ in argon. The MWNTs/magnesium alloy mixtures were hot pressed (823 K, pressure $25.5 \mathrm{MPa}$ ) in a molder under vacuum below $10 \mathrm{~Pa}$. This composite was finally hot extruded 
(723 K, extrusion ratio 9:1) into a $6 \mathrm{~mm} \phi$ rod, and solution treated at $683 \mathrm{~K}$ for 15 minute and aged at $473 \mathrm{~K}$ for 15 hrs according to H5203 of Japan Industrial Standards.

In order to evaluate the corrosion resistance of magnesium alloys rod containing 0,1 and 5 wt\% MWNTs, all samples were trimmed to shorter pieces, and embedded in a resin block with the cross section of the rods appearing in one side. The face of resin block with the cross section of the rod was then wet polished with 4000 mesh polisher and immediately dried. For the wetting test, $2 \mu \mathrm{l}$ of pure water ( $\mathrm{pH}$ 7.7) was dropped using a micro-syringe on the surface of the rods. We have then measured the wetting angle, wetting area, and corrosion resistivity. Corrosion test in salt water was performed by immersing magnesium alloy rods containing 0,1 , and $5 \mathrm{wt} \%$ MWNTs in the salt water ( $3 \mathrm{wt} \% \mathrm{NaCl}, 293 \mathrm{~K}$ ). We have measured the weight loss of the rods for 20 hrs. Corrosion current measurement was performed by using the MWNTs/ magnesium alloy (surface area $\sim 2.0 \mathrm{~cm}^{2}$, polished to remove oxidation layer) as an anode and copper plate (surface area $\sim 1.8 \mathrm{~cm}^{2}$, activated with hydrochloric acid) as a cathode. The electrodes (separation $10 \mathrm{~mm}$ ) were dipped in a water bath filled with $500 \mathrm{ml}$ tap water (pH 8.05, $293 \mathrm{~K})$ with circulating convection. The corrosion current was measured with a digital multimeter (EA707CB-25) for 24 hrs.

First, we performed the wetting tests and found that the surface of the alloy becomes more hydrophobic by increasing the amount of MWNTs as shown in Fig. 1. Wetting angle measurement also showed that water repellency improves by increasing the amount of MWNTs. This is because the number of MWNTs (known to be hydrophobic), mainly appearing on the surface of the alloy, increase accordingly as the nanotube content in the alloy increases. Five minutes after a droplet of water was dropped on the surface of the alloy (Fig. 1), we were able to find under the optical microscope a great deal of bubbles coming out from the pristine sample (see short red arrow in Fig. 1 (a)), showing that magnesium is heavily reacting with water. The alloy containing 1 wt\% of MWNTs (Fig. 1 (b)) was also found to react with water showing the formation of numerous bubbles. However, for the $5 \mathrm{wt} \%$ MWNT/magnesium alloy (Fig. 1 (c)), we were unable to find any bubble, thus indicating that the surface of the sample is much less reactive when compared to the pristine alloy and the one containing $1 \mathrm{wt} \%$ of MWNTs. It is noteworthy that even after 20 hrs, the water droplet attacked the surface of the pristine magnesium alloy, and we could still find bubbles coming out continuously from the material (indicated by short blue arrows in Fig. 1 (a)). Interestingly, we could not find any bubbles coming out from the alloys containing 1 and $5 \mathrm{wt} \%$ of MWNTs. We believe that the presence of a wide vermicular texture in the alloy containing $0 \mathrm{wt} \%$ of MWNTs resulted in a greater water penetration depth into the sample, which was the cause of still having the bubbles after 20 hrs. Vertical section of the samples clearly demonstrates such penetration of the corrosion (as 
indicated by black arrow in Fig. 1(a)). This was the reason why the bubbles were still coming out even after 20 hrs. In the $1 \mathrm{wt} \%$ MWNTs sample, the surface was corroded but no pitting was found inside the sample. For the $5 \mathrm{wt} \%$ MWNTs sample, slight darkening was found down to some depth, suggesting the formation of the stable inert layer (or oxide layer), and no trace of corrosion was found inside the sample. The results shown above suggest that the incorporation of MWNTs into magnesium alloys has extensively improved the corrosion resistance.

By immersing all the samples in salt water ( $3 \mathrm{wt} \% \mathrm{NaCl}$ solution) in order to accelerate the corrosion, we have plotted the weight loss ratio as a function of the immersing time of the samples (Fig. 2). After 20 hrs, the pristine magnesium alloy was dissolve up to $13 \%$ in the salt water, whereas the composites containing 1 and $5 \mathrm{wt} \%$ of MWNTs kept most of its original weight below $2 \%$. Corrosion current measurement using tap water showed that for the $5 \mathrm{wt} \%$ MWNT sample, corrosion current completely stops after 4 hrs (Fig. 3). On the other hand, the corrosion current of the $0 \mathrm{wt} \%$ MWNT sample was almost the same throughout the measurement period. This shows that once the surface of MWNT/magnesium alloy composite is covered with oxide layers, electrochemical reaction stops, but it is not the case for the $0 \mathrm{wt} \%$ of MNWT sample where continuous detachment of oxide layer occurs.

It is clear that the incorporation of MWNTs into magnesium alloys greatly enhances the corrosion resistance. From the experimental results, FE-SEM observations, and elemental analysis, we could propose two main scenarios explaining the corrosion resistivity of magnesium alloy: (1) from the wetting results, we consider that the hydrophobic character of the MWNTs is playing an important role in this high corrosion resistivity. Since corrosion occurs where excess amount of water is in contact with the alloy surface, if the water is repelled from the alloy surface, corrosion is less likely to occur. Therefore, MWNTs mixed with magnesium alloys improve water repellency of the alloy, and leads to the improvement of corrosion resistivity. (2) The reinforcement of the oxide layer with MWNTs was found by the elemental mapping. In the case of magnesium alloy without MWNTs, the oxide layer keeps on coming off from the surface after it is formed. However, when MWNTs are incorporated into the magnesium alloy, the tubes keep the oxide layer from detaching from the alloy, which slows further formation of oxide layer and therefore leads to the large improvement of corrosion resistivity. Fe-SEM observations of the reacted surface showed that the pristine magnesium alloy had more cracks on the surface, when compared to the $5 \mathrm{wt} \%$ MWNTs sample that had fewer cracks. This suggests that the reinforcement of the oxide layer by the presence of MWNTs is taking place in the sample with MWNTs (Fig. 4).

We have shown that the incorporation of MWNTs into magnesium alloys results in a significant enhancement of the corrosion resistivity. Since the inclusion of MWNTs improves 
the mechanical properties of magnesium alloy as well ${ }^{11}$, optimizing the MWNTs dispersion, type, length and diameter of MWNTs used in MWNTs/magnesium alloy composite, would further improve the corrosion resistivity and high mechanical properties at the same time. Our results could be considered as a breakthrough and will develop applications of a light metal such as magnesium, which, by an innovative process of incorporating MWNTs, becomes mechanically strong and anticorrosive. Therefore, light and strong metal composites reinforced with MWNTs could be expected to replace, in some cases, steel and plastics due to their anti-corrosive properties, performances and recyclability.

This work was supported by the CLUSTER (the second stage) of Ministry of Education, Culture, Sports, Science and Technology of Japan and a Grant-in-Aid from the Ministry of Education, Culture, Sports, Science and Technology of Japan (Nos. 19002007, 18710084 and 1771096). 


\section{References}

1. D.I. Proskurovsky, V.P. Rotshtein, G.E. Ozur, Y.F. Ivanov, A.B. Markov, Surf. Coat. Technol. 125, 49 (2000).

2. F. Mansfeld, S.L. Jeanjaquet, Corrosion Science 26, 727 (1986).

3. P.P. Trzaskoma, Corrosion 42, 609 (1986).

4. United States Patent No 5494634 (1996)

5. G. Yamaguchi, M. Mino, J. Seki, E. Sakita, Y. Miyata and K. Arita, Advanced Composite Materials 1, 3-10 (1991)

6. P.P. Trzaskoma, Corrosion 42, 609 (1986).

7. J. Yang, R. Schaller, Mater. Sci. Eng. A 370, 512 (2004).

8. E. Carreno-Morelli, J. Yang, E. Couteau, K. Hernadi, J.W. Seo, C. Bonjour, L. Forro, R. Schaller, Phys. Stat. Sol. 201, R53 (2004).

9. C.S. Goh, J. Wei, L.C. Lee, M. Gupta, Mater. Sci. Eng. A 423, 153 (2006).

10. C.S. Goh, J. Wei, L.C. Lee, M. Gupta, Nanotechnology 17, 7 (2006).

11. Y. Shimizu, S. Miki, T. Soga, I. Itoh, H. Todoroki, K. Sakaki, T. Hosono, Y.A. Kim, T. Hayashi, M. Endo, Scripta Materialia 58, 267 (2008).

12. M. Endo, Chem. Tech. 568 (1988).

13. A. Oberlin, M. Endo, T. Koyama, J. Crys. Grow. 32, 335 (1976). 


\section{Figure captions}

Figure 1 Wetting and corrosion test using pure water droplet for the pristine (a), $1 \mathrm{wt} \%$ (b) and 5 wt\% (c) MWNTs-reinforced magnesium alloy composites. Left row shows the samples (6 mm diameter) after 5 minutes, and middle row shows the samples after 20 hours. Difference in the number of bubbles among the samples can be clearly seen as bright spots (as indicated by short red arrow). Wetting angles have changed from $20^{\circ}$ to $32^{\circ}$ for pristine and $5 \mathrm{wt} \%$ MWNTs-reinforced magnesium alloy composites. After $20 \mathrm{hrs,} \mathrm{the} \mathrm{surfaces} \mathrm{are} \mathrm{similarly}$ corroded, but in the pristine magnesium alloy (a), bubbles were still coming out (short blue arrow). Right column shows the optical micrograph of the vertical section that was obtained by cutting the samples along the dotted line shown in the middle images. Green dotted line shows the edge between the section and the surface of the composite. Black arrow shows the pitting formed as worm-eaten holes.

Figure 2 Weight loss ratio of pristine and MWNTs-reinforced magnesium alloy composites in 3 wt\% salt water.

Figure 3 Corrosion current of pristine and MWNTs-reinforced magnesium alloy composites in tap water $\left(20^{\circ} \mathrm{C}, \mathrm{pH} 8.05\right)$.

Figure 4 FE-SEM images exhibiting the surface morphologies of the pristine and 5 wt\% MWNTs-reinforced magnesium alloy composites, respectively. The pristine magnesium alloy has more cracks compared to $5 \mathrm{wt} \%$ MWNTs/magnesium alloy composite. As shown in the right image, we can find the MWNT reinforcing the oxide layer. 


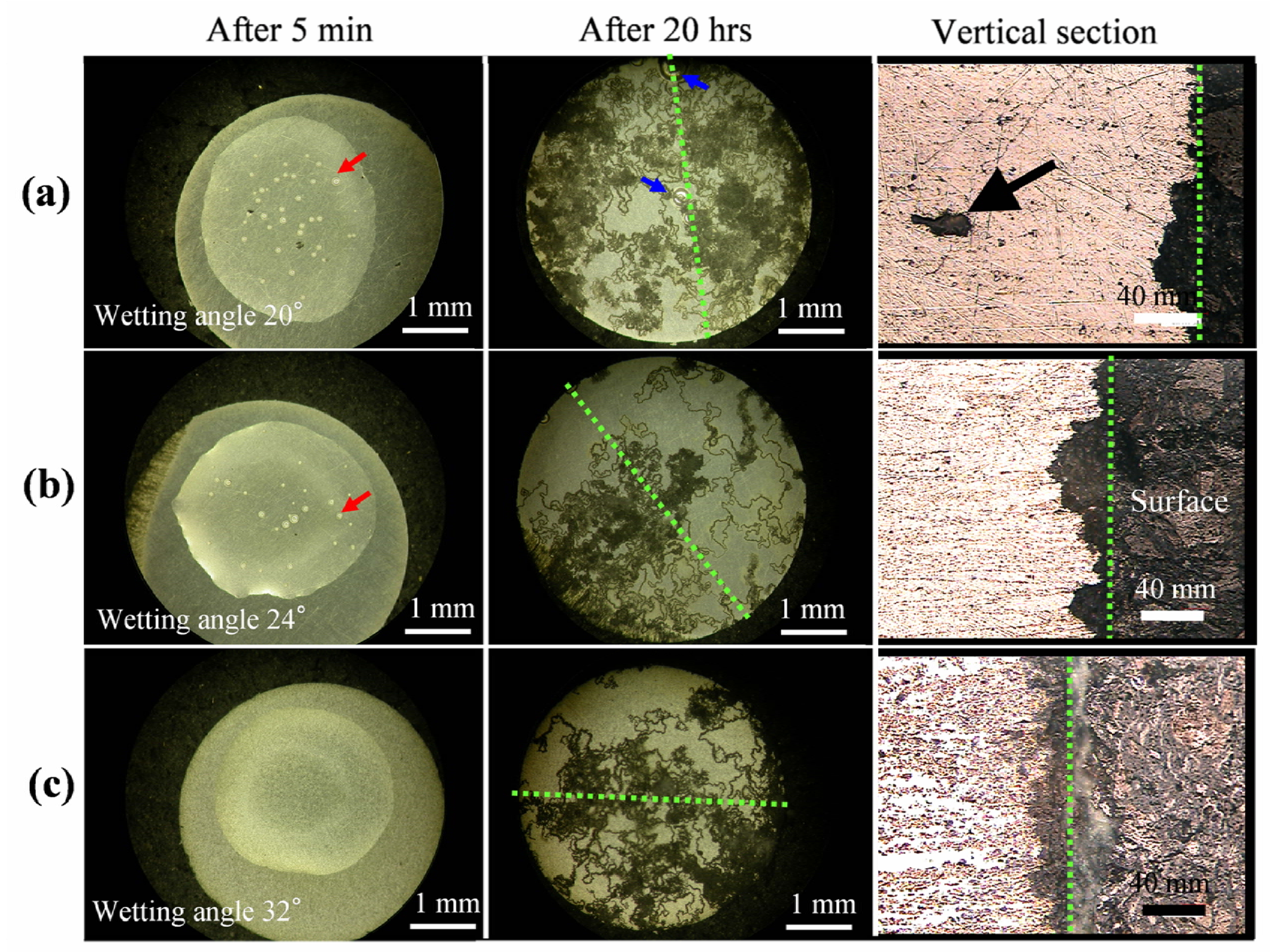

Figure 1 


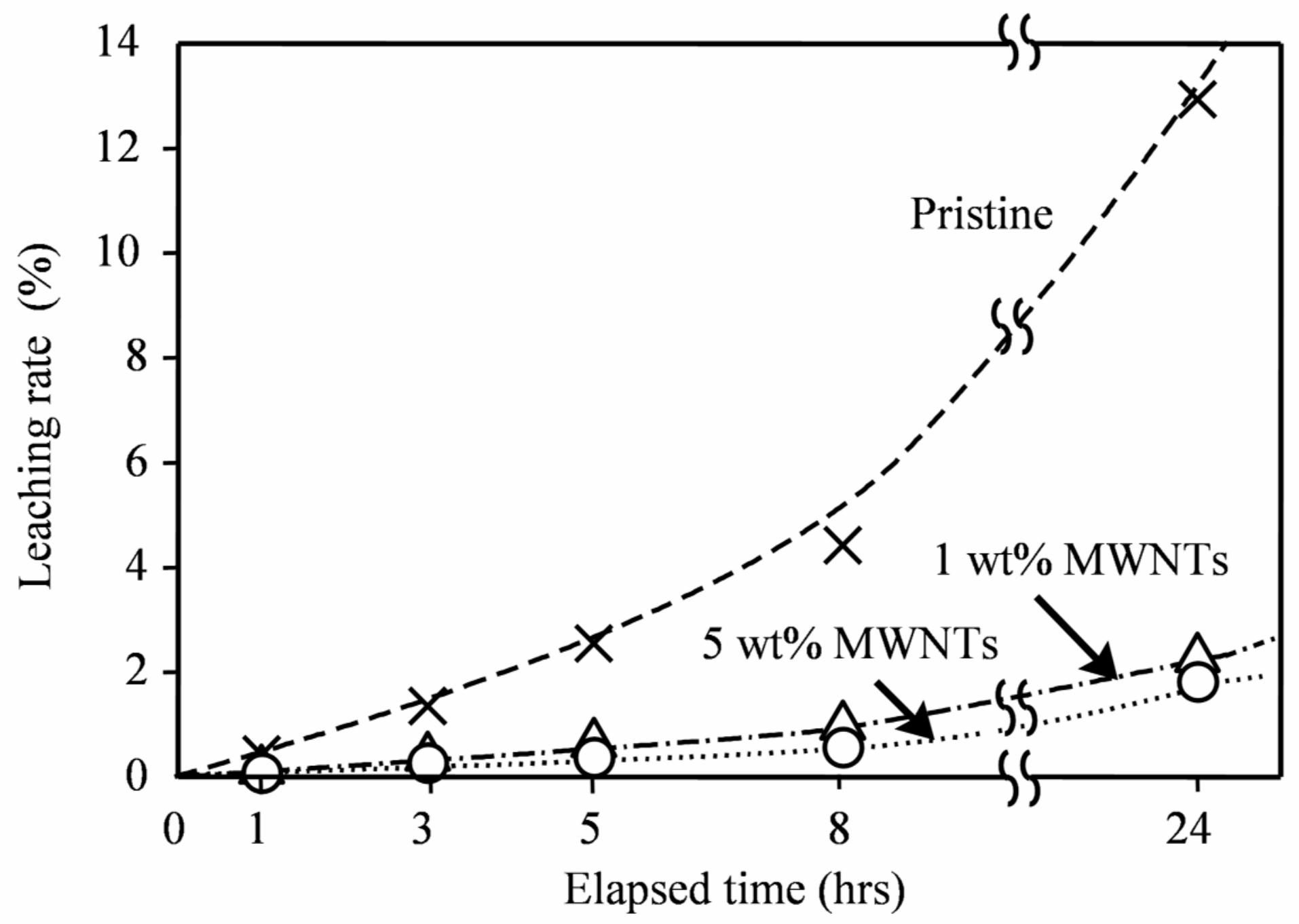

Figure 2 


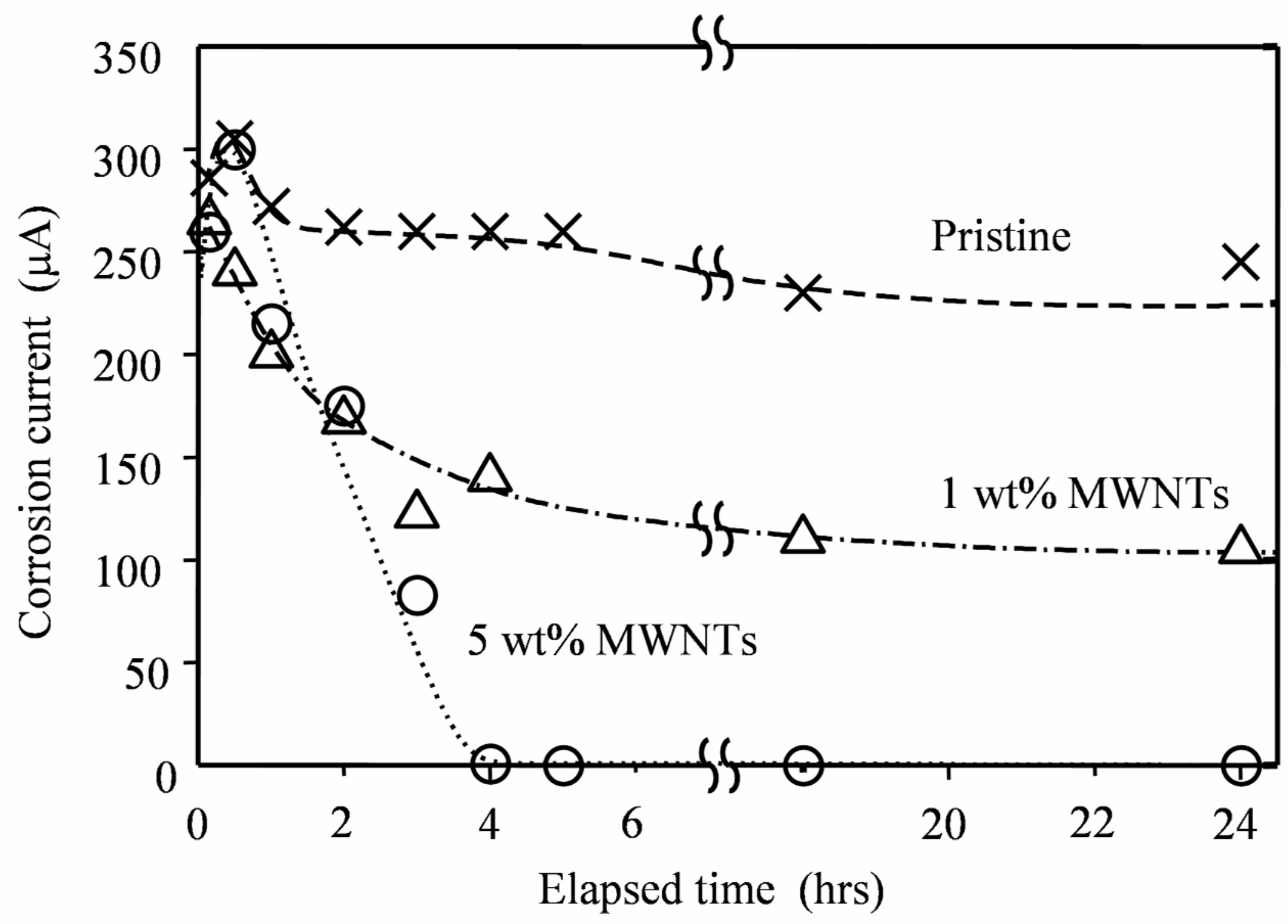

Figure 3 


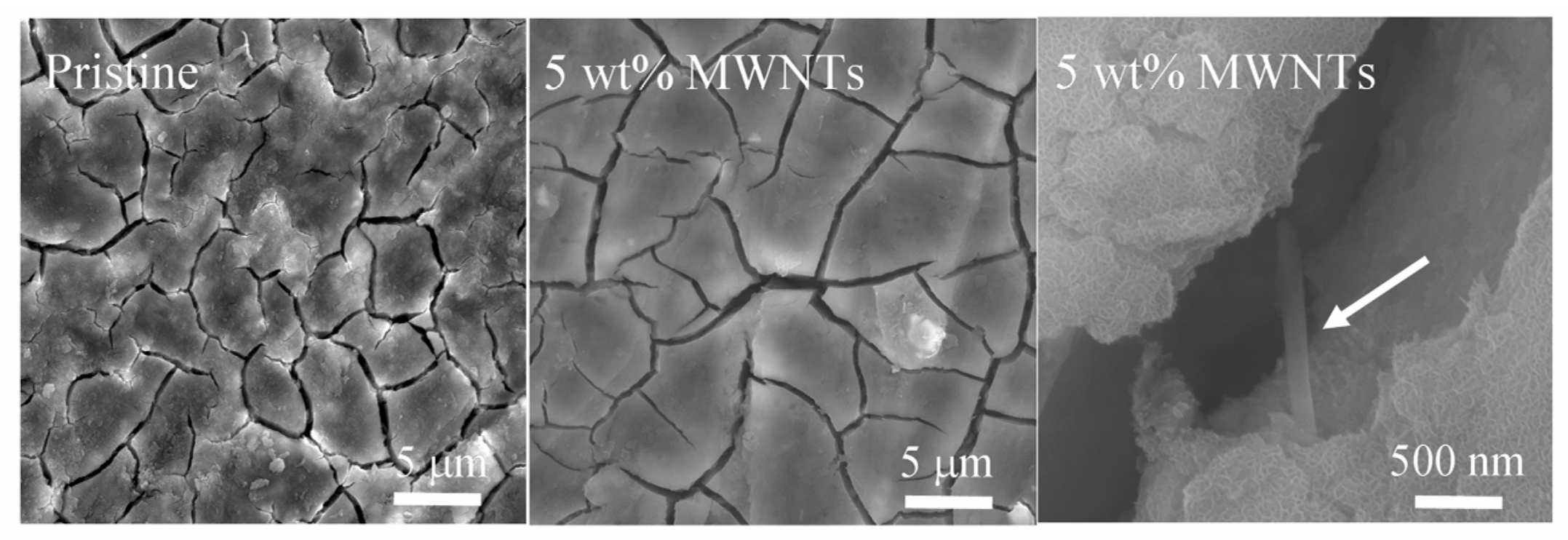

Figure 4 